\title{
COLABORACIÓN ESPECIAL
}

\section{FUNCIONAMIENTO Y DISCAPACIDAD: LA CLASIFICACION INTERNACIONAL DEL FUNCIONAMIENTO (CIF)}

\author{
Juan Antonio Fernández-López (1), María Fernández-Fidalgo (2), Reed Geoffrey (3) Gerold \\ Stucki $(4,5,6)$ y Alarcos Cieza $(5,6,7)$.
}

(1) Centro de Salud de Riosa,. Servicio de Salud del Principado de Asturias (SESPA). España.

(2) Facultad de Psicología, Universidad de Oviedo. España.

(3) International Union of Psychological Science. Madrid. España.

(4) Seminar of Health Sciences and Health Policy, University of Lucerne, and at SPF, Nottwil. Switzerland.

(5) ICF Research Branch, WHO FIC CC Germany (DIMDI) at SPF, Nottwil, Switzerland and at IHRS, LudwigMaximilians-University, Munich, Germany.

(6) Swiss Paraplegic Research (SPF), Nottwil, Switzerland.

(7) Institute for Health and Rehabilitation Sciences (IHRS), Unit for Biopsychosocial Health. Ludwig-MaximiliansUniversity. Munich Germany.

\section{RESUMEN}

La Clasificación Internacional del Funcionamiento, de la Discapacidad y de la Salud (CIF) constituye el marco conceptual de la OMS para una nueva comprensión del funcionamiento, la discapacidad y la salud. Es una clasificación universal que establece un marco y lenguaje estandarizados para describir la salud y las dimensiones relacionadas con ella. Abarca tres componentes esenciales: funciones corporales/estructuras, actividad y participación, integrados bajo los términos "funcionamiento" y "discapacidad", que dependen de la condición de salud y de su interacción con factores contextuales. Los componentes están clasificados mediante categorías. Hoy por hoy consta de 1.424 categorías organizadas en una estructura jerárquica de 4 niveles de menos preciso a más. En el caso de los factores contextuales la cuantificación de las categorías mediante calificadores establece en qué medida un factor actúa como barrera o facilitador.

La instrumentalización de la CIF con fines prácticos ha llevado al desarrollo de grupos de categorías (núcleos básicos) útiles para la clínica práctica, la provisión de servicios o la investigación. Los núcleos básicos son abreviados o extensos según la intención sea estudiar condiciones específicas o realizar aplicaciones integrales multidisciplinares. Existen núcleos básicos para enfermedades muy prevalentes según la fase del proceso: aguda, postaguda, crónica o comunitaria, cubriendo todo el proceso de enfermedad y toda la cadena sanitaria. Su aplicación clínica mejora la comunicación médico-paciente y el establecimiento de objetivos terapéuticos integrales. Asimismo, sirve para la evaluación multidisciplinar en medios clínicos, legales y de programas. La CIF logra la superación de conceptos de amplia utilización sanitaria, como bienestar, estado de salud o calidad de vida relacionada con la salud y abre una nueva línea de enseñanza estudiantil en todas las especialidades y de formación integral multiprofesional. Palabras clave: Salud. Clasificación, CIF. Discapacidad.

\section{Correspondencia:}

Juan Antonio Fernández-López

Centro de Salud de Riosa

Servicio de Salud del Principado de Asturias (SESPA)

33160 Asturias, España. Tel/Fax: 985766293

Correo electrónico: juanantonio.fernandez@ sespa.princast.es

\section{ABSTRACT \\ Functioning and Disability: The International Classification of Functioning, Disability and Health (ICF)}

The World Health Organization's International Classification of Functioning, Disability and Health (ICF) has provided a new foundation for our understanding of health, functioning, and disability. It covers most of the health and health-related domains that make up the human experience, and the most environmental factors that influence that experience of functioning and disability. With the exhaustive ICF, patients' functioning -including its components body functions and structures and activities and participation-, becomes a central perspective in medicine. To implement the ICF in medicine and other fields, practical tools (= ICF Core Sets) have been developed. They are selected sets of categories out of the whole classification which serve as minimal standards for the assessment and reporting of functioning and health for clinical studies and clinical encounters (Brief ICF Core Set) or as standards for multiprofessional comprehensive assessment (Comprehensive ICF Core Set). Different from generic and condition-specific health-status measures, the ICF Core Sets include important body functions and structures and contextual factors. The use of the ICF Core Sets provides an important step towards improved communications between healthcare providers and professionals, and will enable patients and their families to understand and communicate with health professionals about their functioning and treatment goals. Specific applications include multiand interdisciplinary assessment in clinical settings and in legal expert evaluations and use in disease or functioning-management programs. The ICF has also a potential as a conceptual framework to clarify an interrelated universe of health-related concepts which can be elucidated based on the ICF and therefore will be an ideal tool for teaching students in all medical fields and may open doors to multi-professional learning.

Key words: Health. Classification. ICF. Disability. 


\section{INTRODUCCIÓN}

La Clasificación Internacional del Funcionamiento, de la Discapacidad y de la Salud aprobada en 2001, más comúnmente conocida como CIF, constituye el marco conceptual de la OMS para una nueva comprensión del funcionamiento, la discapacidad y la salud ${ }^{1}$. Se trata de una clasificación universal útil para múltiples usos y sectores que pretende establecer un marco y un lenguaje estándar para describir la salud y las dimensiones relacionadas con ella. Se la denomina habitualmente CIF porque pone el acento más en la salud y el funcionamiento que en la discapacidad, entendiendo funcionamiento como lo referente a las funciones corporales, las actividades y la participación y la discapacidad como lo relativo a deficiencias, limitación de actividades o restricción de la participación. Es, pues, una perspectiva corporal, individual y social. Adicionalmente la CIF también considera los factores contextuales (ambientales y personales) que interaccionan con estos componentes.

Tradicionalmente la salud y la discapacidad se han definido como conceptos excluyentes. Así, la discapacidad se entendía como un estado que empieza donde termina la salud, pasando entonces a constituir una categoría separada (modelo biomédico). En este punto la CIF supone un cambio conceptual radicalmente distinto. Asume que todos podemos experimentar en un momento determinado de nuestra vida un deterioro de la salud y, por tanto, un cierto grado de discapacidad. Así, salud y discapacidad se extienden por igual a lo largo del continuum de nuestra vida y de todas sus facetas y no son, por tanto, categorías separadas. Discapacidad no es, pues, la característica de algunos grupos sociales sino que se trata de una experiencia humana universal, un concepto dinámico bidireccional fruto de la interacción entre estado de salud y factores contextuales (modelo biopsicosocial) $)^{2}$.
Para alcanzar este nuevo nivel de comprensión bastó con hacer virar el punto de atención desde la causa hacia el impacto. Con ello todos los estados de salud se colocaron en un mismo nivel, permitiendo su comparación mediante una métrica común. Por eso la CIF es un lenguaje común a todas las personas, a lo largo de toda la vida y que abarca por igual a todos los aspectos de la misma. Esto fue razón suficiente para su buena acogida no sólo por parte de los profesionales sanitarios y científicos sino también por los propios pacientes, que ven reforzada su perspectiva en el sistema sanitario.

La CIF pertenece a la Familia Internacional de Clasificaciones de la OMS (FICOMS). Su miembro más conocido es la CIE-10 (Clasificación Internacional de Enfermedades) $)^{3}$. La información de la CIE10 resulta enriquecida por la obtenida con la CIF -en lo que se refiere al funcionamiento- constituyendo en conjunto la familia de las clasificaciones de la OMS y proporcionando una visión más amplia y significativa de la salud de personas y poblaciones.

\section{LA CIF EN LA PERSPECTIVA DE LA OMS Y DE LA ONU}

La CIF, que actualmente es coordinada por el equipo de la Clasificación, Terminología y Estándares (CTS) de la OMS, sirve como marco de referencia para toda la OMS. Fundamentalmente es el marco de referencia del equipo de Discapacidades y Rehabilitación (DAR), bajo la dirección del Departamento de Prevención de la Violencia, Daños y Discapacidad.

A la vez que la CIF fue desarrollada por la OMS, agencia especializada responsable de salud dentro del sistema de las Naciones Unidas (ONU), fue aceptada por ella como clasificación social y actualmente sirve de marco de referencia para 
las Naciones Unidas y otras agencias especializadas como la División Estadística de la ONU (UNSTAT), la Organización Educativa, Científica y Cultural de la ONU (UNESCO) y la Organización Internacional del Trabajo (ICO).

El modelo de entendimiento del funcionamiento humano como una experiencia universal -según el modelo CIF-, es también la base de caracterización de la discapacidad de la Convención de Derechos de las Personas Discapacitadas de la ONU $^{4}$ aprobada en 2006 en Nueva York. Define las obligaciones de los Estados de promover, proteger y asegurar los derechos de las personas discapacitadas y propone los pasos que se deben dar para adoptar los cambios medioambientales necesarios.

La primera versión de la CIF se publicó en 2001. Al igual que la CIE experimentará actualizaciones futuras y un proceso de revisión final. La actualización la realiza el equipo CTS de la OMS en colaboración con el Grupo de Referencia del Funcionamiento y la Discapacidad (FDRG), de la Red de Centros Colaboradores para la Familia Internacional de las Clasificaciones (WHO FIC CC Network). La actualización incluye información procedente de un amplio rango de estudios de comprobación y validación dirigidos en colaboración con la FDRG. En el futuro la CIF puede evolucionar hacia una clasificación similar a la tomada por la Nomenclatura Sistematizada de la Medicina (Snomed). ${ }^{5}$

\section{MODELO CONCEPTUAL DE LA CIF}

Como se muestra en la figura 1, la CIF está basada en un modelo integral del funcionamiento, la discapacidad y la salud. Consta de tres componentes esenciales. El primero de ellos, funciones y estructuras corporales, tiene que ver con las funciones fisiológicas/psicológicas y los elementos anatómicos, y es su ausencia o alteración lo que concebimos como deficiencias en las funciones y las estructuras. El segundo componente, la actividad, se refiere a la ejecución individual de tareas y las dificultades que tiene una persona para realizarlas son las limitaciones. El tercer componente, la participación, se refiere al desenvolvimiento de las situaciones sociales y los problemas que el individuo experimenta en tal desenvolvimiento constituyen las restricciones ${ }^{6}$.

Los tres componentes están integrados bajo los términos funcionamiento y discapacidad y dependen tanto de la condición

Figura 1

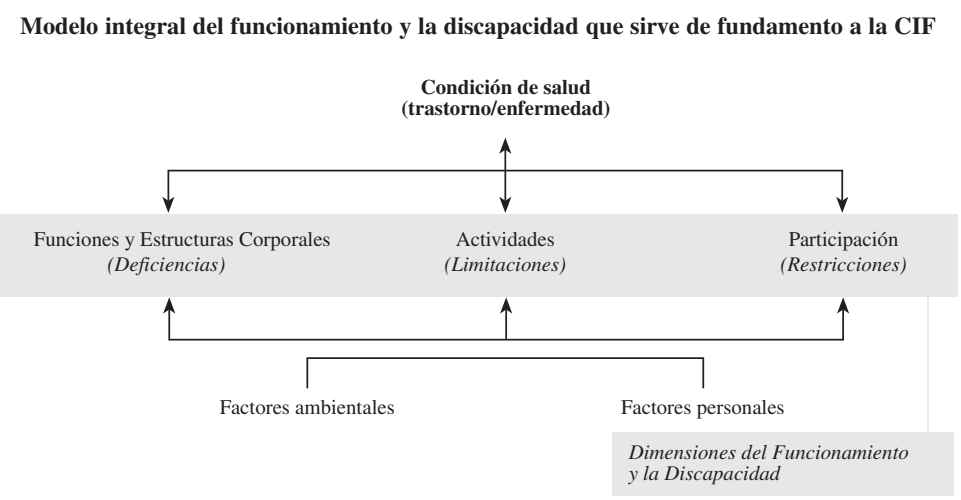


de salud como de su interacción con los factores personales y ambientales. Discapacidad es el término genérico que incluye déficits, limitaciones y restricciones e indica los aspectos negativos de la interacción entre el individuo (con una condición de salud dada) y sus factores contextuales (factores ambientales y personales). Por el contrario, funcionamiento es el término genérico que incluye función, actividad y participación, indicando los aspectos positivos de esa interacción. Son las dos caras de una misma moneda dependiente de la condición de salud y de la influencia de los factores contextuales.

Los factores contextuales pueden ser externos (ambientales) o internos (personales). Ambientales son las actitudes sociales, las características arquitectónicas, el clima, la geografía, las estructuras legales y sociales, etc. Personales son la edad, el sexo, la biografía personal, la educación, la profesión, los esquemas globales de comportamiento, el estilo coping, el carácter, etc.

El impacto de los factores contextuales es tan importante que respecto al funcionamiento pueden actuar como facilitadores o como barreras. Hasta la fecha se han podido clasificar los factores ambientales mientras que los factores personales están en vía de serlo.

\section{ESTRUCTURA OPERACIONAL DE LA CIF}

Desde la visión integral de este modelo, el nivel personal de funcionamiento es algo complejo con múltiples determinantes e interacciones que ejercen sus efectos a múltiples niveles e incluyen diferentes dimensiones $^{7}$. Los componentes de las funciones y estructuras corporales, las actividades, la participación y los factores ambientales están clasificados en la CIF mediante categorías. Sólo los factores personales permanecen, por el momento, sin clasificar. Es de esperar que en los próximos años los esfuerzos de la FDRG culminen en el desarrollo de una clasificación de factores personales -estudiando la posibilidad y la metodología necesaria-. Hoy por hoy la CIF consta de 1.424 categorías mutuamente excluyentes que tomadas en conjunto cubren un espectro exhaustivo e integral de la experiencia humana y que están organizadas como una estructura jerárquica de 4 niveles diferenciados de menor a mayor precisión. (figura 2).

Las categorías de la CIF están indicadas por medio de códigos alfanuméricos con los cuales es posible clasificar funcionamiento y discapacidad, tanto a nivel individual como poblacional. Un ejemplo de la estructura jerárquica es el siguiente:

Figura 2

Estructura y distribución de las $\mathbf{1 . 4 2 4}$ categorías de la CIF en sus cuatro componentes y niveles jerárquicos

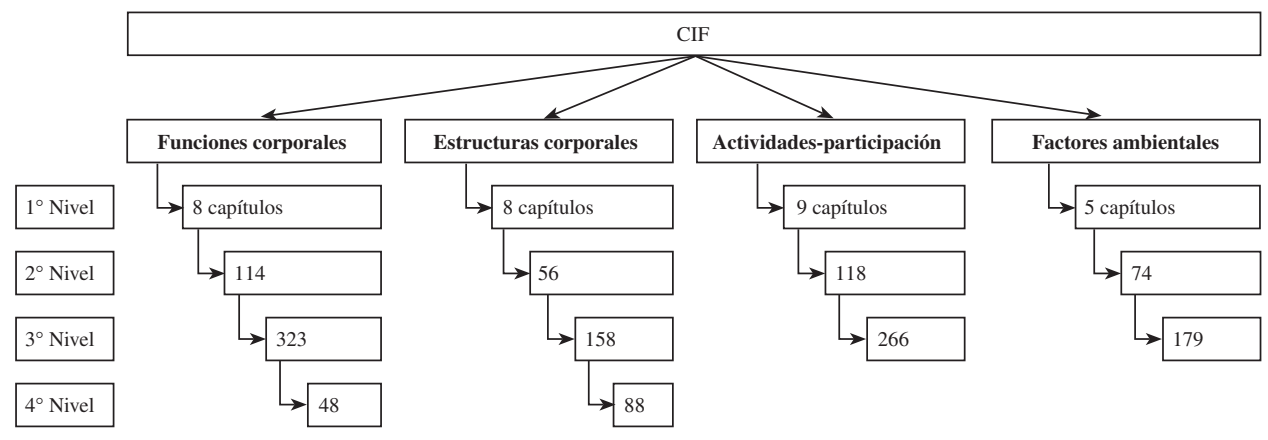




\begin{tabular}{|l|l|l|}
\hline Capítulo/ $1^{\circ}$ Nivel & b2 & Funciones sensoriales y dolor \\
\hline $2^{\circ}$ nivel & b280 & Sensación de dolor \\
\hline $3^{\circ}$ nivel & b2801 & Dolor en una parte del cuerpo \\
\hline $4^{\circ}$ nivel & b28013 & Dolor de espalda \\
\hline
\end{tabular}

Según esta estructura jerárquica la categoría de nivel más alto $\left(4^{\circ}\right)$ comparte los atributos de la categoría de menor nivel $\left(1^{\circ}\right)$ a la cual pertenece. Así, en nuestro ejemplo, el uso de una categoría de más nivel (b28013, Dolor de espalda) implica automáticamente que es aplicable a una categoría de nivel inferior (b2801, Dolor en una parte del cuerpo).

Dado que las categorías CIF están siempre acompañadas de una breve definición -con inclusiones y exclusiones- toda la información acerca del funcionamiento se muestra de forma clara e inequívoca, tanto general como detalladamente, según usemos el $2^{\circ}, 3^{\circ}$ y $4^{\circ}$ nivel de categorías.

Para cuantificar la magnitud de un problema en las diferentes categorías CIF se usan los calificadores CIF, los cuales son descriptores de calidad ponderados matemáticamente que registran la presencia o severidad de un problema a nivel corporal, personal o social. Así, un problema puede suponer un deterioro, una limitación o una restricción que puede calificarse desde 0 (no problema: 0-4\%), 1 (problema leve: 524\%), 2 (problema moderado: 25-49\%), 3 (problema severo: 50-95\%) hasta 4 (problema total: $96-100 \%$ ).

Por su parte, los factores ambientales son cuantificados con una escala negativa o positiva que indica la medida en la cual un factor ambiental actúa como barrera o facilitador $^{1}$ :

\begin{tabular}{|l|l|}
\hline $0-$ No barrera & $0-$ No facilitador \\
\hline$-1-$ Barrera Leve & $+1-$ Facilitador Leve \\
\hline$-2-$ Barrera Moderada & $2-$ Facilitador Moderado \\
\hline$-3-$ Barrera Severa & $+3-$ Facilitador Severo \\
\hline$-4-$ Barrera Completa & $+4-$ Facilitador Completo \\
\hline
\end{tabular}

\section{DESARROLLOS METODOLÓGICOS}

Con la estructura operativa descrita más arriba los componentes del modelo CIF -excepto los factores personalesquedan clasificados de modo que nos proporcionan una comprensión estandarizada y una descripción integral de la salud y de los estados relacionados con ella. Ahora bien, un compendio integral de la clasificación al completo resulta poco práctico en la clínica diaria, a menos que lo transformemos en una herramienta más manejable ${ }^{8-9}$.

Para facilitar tal descripción sistemática y completa del funcionamiento y su uso en la clínica diaria se ha puesto en marcha un proyecto cuyo objetivo es desarrollar grupos de categorías CIF útiles para la clínica práctica, la provisión de servicios y la investigación, así como para vincular la CIF a las condiciones de salud codificadas con la $\mathrm{CIE}^{10}$. Con este fin el grupo de investigación de la CIF del centro colaborador de la OMS en la Universidad Ludwig Maximilian, en Munich, junto al grupo de Clasificación, Evaluación, Encuestas y Terminología de la OMS (CAS) y otras organizaciones participantes, ha desarrollado los llamados núcleos básicos de la CIF (ICF Core Sets).

Los núcleos básicos CIF son listas consensuadas de categorías relevantes de la CIF que cuando son específicas para enfermedades concretas o para contextos sanitarios se denominan núcleos básicos abreviados (Brief ICF Core Sets), y pueden usarse en estudios clínicos o estadísticas sanitarias. Consisten en el menor número necesario de categorías CIF para describir un problema prototípico de funcionamiento y salud en pacientes con una determinada/específica condición ${ }^{11}$. Por el contrario, para cuando necesitamos realizar una evaluación integral multidisciplinaria y multiprofesional se han desarrollado listados más extensos de categorías 
relevantes. Son los llamados núcleos básicos extensos (Comprehensive ICF Core Sets $)^{12}$.

En ambos casos se listan las categorías CIF relevantes que pueden ser medidas, si bien no indican cómo deben medirse ${ }^{6}$. Existen disponibles núcleos básicos CIF para episodios agudos cardiopulmonares, musculoesqueléticos o neurológicos para el tratamiento agudo en hospitales ${ }^{13-14}$. Asimismo, se han desarrollado hasta la fecha 20 núcleos básicos CIF para condiciones postagudas y crónicas de las enfermedades más prevalentes y su aplicación en distintos contextos clínicos: postagudos, crónicos, rehabilitadores y comunitarios. De aquí resulta que los núcleos CIF pueden usarse a lo largo de todo el proceso de enfermedad y en toda la cadena sanitaria.

En contraste con las medidas existentes del estado de salud -genéricas y/o específicas- los CIF core sets no incluyen sólo personas con una enfermedad determinada, sino que al considerar funciones y estructuras corporales así como factores contextuales proporcionan una visión más completa e integral de su funcionamiento.

Los núcleos básicos desarrollados hasta la fecha han de ser comprobados y validados en distintas lenguas, medios culturales, grupos de pacientes y de profesionales. Posteriormente han de ser aprobados y recomendados para su uso general por un panel de expertos internacionales a la vista del proceso previo de validación.

\section{IMPLICACIONES SANITARIAS}

Aunque la aplicación del modelo biopsicosocial en la medicina ya se viene aplicando desde hace tiempo en la rehabilitación física y mental ${ }^{15-16}$, la CIF ofrece el modelo científico válido universalmente para ejecutar el cambio efectivo desde el paradigma conceptual puramente médico hacia un modelo biopsicosocial del funcionamiento humano y la discapacidad.

$\mathrm{El}$ reconocimiento del funcionamiento y la discapacidad como problema mayor de salud pública, tanto en países desarrollados como emergentes, es lo que ha llevado a la OMS a desarrollar la CIF como lenguaje internacional estandarizado capaz de describir y clasificar la salud y las dimensiones relacionadas con ella, y así promover un marco común para la medición de los resultados sanitarios.

La CIF complementa los indicadores que tradicionalmente se han utilizado para medir la mortalidad o las enfermedades ${ }^{3}$. Esta complementariedad se hizo necesaria porque tales datos no recogen adecuadamente los resultados de salud en individuos o poblaciones (por ejemplo, los diagnósticos por sí solos no explican lo que son capaces de hacer las personas que padecen las enfermedades, cuál es su pronóstico, qué necesitan o cuál es el coste de sus tratamientos) ${ }^{8-9}$. En este sentido la CIF es útil para evaluar el diferencial existente entre el funcionamiento real y el potencial del enfermo (con ayuda de los calificadores). Asimismo identifica facilitadores y/o barreras ambientales que deben ser objeto de intervención y sirve de guía para la evaluación multidisciplinaria de personas con una determinada patología.

Más específicamente la CIF ha cubierto todo el espectro de problemas que acontecen en un amplio rango de enfermedades y a lo largo de toda la cadena de cuidados médicos. En la clínica práctica diaria se han publicado diversos trabajos aplicando la CIF en contextos clínicos de atención primaria, enfermería, logopedia o terapia ocupacional, mostrando su utilidad en el trabajo diario y en la evaluación en ensayos clínicos ${ }^{6,10,14,17}$. También se presenta muy útil (para personas discapacitadas y profesionales) en la evaluación de los medios sanitarios que atienden a pacientes 
crónicos o con discapacidad como centros de rehabilitación, unidades de enfermería, instituciones psiquiátricas y servicios sociales.

El uso de categorías CIF en la descripción del funcionamiento de las personas ofrece una excelente oportunidad para mejorar la comunicación médico-paciente acerca del nivel de función y de los objetivos terapéuticos. También es muy útil en la evaluación multidisciplinar del proceso rehabilitador y en las evaluaciones de expertos legales. En algunos países desarrollados ha sido introducida -la CIF y su modelo de discapacidad-, en las políticas legislativas y sociales de diferentes sectores. En este sentido la OMS está promoviendo su uso en servicios como la educación, el trabajo, los seguros o la estadística sanitaria. Se espera que en el futuro se convierta en un estándar mundial para datos de discapacidad y un modelo de política social, favoreciendo la introducción de legislaciones adecuadas en muchos países del mundo.

En nuestro medio se han descrito los fundamentos de la CIF en diversa forma ${ }^{18-}$ 19 y se ha podido demostrar en la práctica un alto grado de equivalencia con otras medidas ampliamente utilizadas en el ámbito sociosanitario para valorar la dependencia -como el Índice de Barthel o de Actividades de la Vida Diaria Básicas- y una mejor clarificación de las áreas de estu$\mathrm{dio}^{20}$.

También diversos estudios han mostrado que ítemes de múltiples instrumentos de medida de la salud pueden ser equiparados a la $\mathrm{CIF}^{21} \mathrm{y}$, más aún, que la CIF representa ampliamente los contenidos de medidas de calidad de vida relacionada con la salud como el SF-36 23 . Esto puede decirse tanto en lo tocante a la extensión de contenidos de la medida como de la profundidad de los distintos niveles de especificación dentro de las dimensiones de la salud o relacionadas con la salud ${ }^{22}$. En este contexto los núcleos básicos CIF pueden considerarse como evidencias o "comprobaciones conceptuales" del estado de salud ${ }^{10,21}$.

La CIF, pues, es un potencial marco conceptual para clarificar y organizar un universo interrelacionado de conceptos que tienen que ver con la salud, como son bienestar, estado de salud, calidad de vida o calidad de vida relacionada con la salud, y contribuye a la comprensión de la salud humana. ${ }^{22-25}$.

El resultado es una estructura conceptual coherente como área de investigación multifacético, en la que los investigadores de diversos campos pueden coincidir conectando sus conocimientos y esfuerzos para mejorar el funcionamiento y la calidad de vida de las personas con discapacidad. Asimismo, es una oportunidad especial para la investigación científica en áreas relacionadas -como la psicología o la sociología-, con el fin de involucrarse en un sector de la investigación que es relevante tanto para la vida de las personas como para la sociedad. ${ }^{26}$ En este sentido la investigación del funcionamiento y la discapacidad ha de partir de una perspectiva interdisciplinaria e ir hacia un objetivo último transdisciplinario que sitúe sus relaciones en un sistema global más allá de las limitaciones inherentes a las disciplinas particulares ${ }^{27,28}$.

Sin embargo, por ahora es difícil esta articulación de intereses. La mayoría de los programas de financiación tienen una clara perspectiva biomédica o una perspectiva exclusiva de alguna disciplina ${ }^{29}$. Un paso importante y necesario para hacer "verdadera" ciencia sería una red paralela de ayudas regionales, nacionales e internacionales con la colaboración de centros universitarios interdisciplinarios ${ }^{30}$. Igualmente importante es crear instituciones de investigación desde la perspectiva integral del funcionamiento humano, dotándolas de la adecuada infraestructura. Un ejemplo práctico 
de ello es el Swiss Paraplegic Research $(S P F)^{31}$. Este instituto de investigación dirige programas en los terrenos científicos que se consideran implicados en el emergente campo de las ciencias del funcionamiento humano, y su misión es "contribuir al óptimo funcionamiento, la integración social y la calidad de vida de las personas con discapacidad a través de la investigación clínica orientada a la comunidad" 32-33.

Futuros centros integrales de este tipo serán los centros de excelencia y nodos de investigación que superen los límites particulares de las ciencias naturales, la ingeniería médica y las ciencias sociales y del comportamiento.

Por otro lado, especialmente en los tiempos que corren de crisis económica global, desde la perspectiva de la economía sanitaria la CIF rinde importantes beneficios al permitir monitorizar y explicar los costes de las discapacidades y de los cuidados sanitarios. Midiendo funcionamiento y discapacidad se puede cuantificar la pérdida de productividad y su impacto en la vida de las personas y de las sociedades. Resulta, pues, de gran utilidad en la evaluación de programas. Finalmente, la CIF y sus núcleos bási$\cos$ son instrumentos ideales para la enseñanza estudiantil en todas las especialidades y abren el camino a una nueva línea de formación continuada multiprofesional.

En resumen, la CIF -como marco de la OMS para la salud y la discapacidad-, es la base conceptual para la definición, medición y formulación de políticas de salud y discapacidad. Abarca la mayoría de los aspectos del funcionamiento, la discapacidad y la salud relevantes para los pacientes y que son objetivos de la intervención sanitaria y social. Asimismo, como base esencial para la estandarización de datos mundiales, relacionados con el funcionamiento y la discapacidad, constituye la clasificación universal de referencia para su uso en el campo de la salud y los sectores relacionados.

\section{BIBLIOGRAFIA}

1. World Health Organization. The International Classification Functioning, Disability and Health. Geneva: WHO; 2001.

2. Bickenbach JE, Chatterji S, badley EM, Ustün TB. Models of disablements, disabilities and handicaps. Soc Sci Med. 1999; 48: 1173-87).

3. Organización Mundial de la Salud. Clasificación Estadística Internacional de Enfermedades, décima edición (CIE-10). 10ª revisión. Ginebra: Organización Mundial de la Salud; 1992.

4. United Nations. Convention on the Rights of Persons with disabilities, 2007. Citado el 7 de Febrero 2009. Disponible en: www.un.org/ disabilities /default.asp?navid=12\&pid=150.

5. World Health Organization. Report for the consultation meeting on the WHO business plan for classifications. Geneva: WHO; 2005.

6. Stuki G. International Classification of Functioning, Disability, and Health (ICF): a promising framework and classification for rehabilitation medicine. Am J Phys Med Rehabil. 2005; 84: 733-40.

7. Waslsh NE. The Walter J. Zeiter lecture. Global initiatives in rehabilitation medicine. Arch Phys Med Rehabil. 2004; 85: 1395-402).

8. Üstün B, Chatterji S, Kostanjsek N. Comments from WHO for the Core Sets. J Rehabil Med. 2004; 36 (suppl44): 7-8.

9. Chamie M. What does morbidity have to do with disability? Disabil Rehabil. 2005; 17: 323-37.

10. Stucki G, Grimby G. Foreword: Applying the ICF in Medicine. J Rehabil Med. 2004; 44 (suppl): 5-6.

11. Weigl M, Cieza A, Andersen C, Kollerits B, Amann E, Stucki G. Identification of relevant ICF categories in patients with chronic health conditions: a Delphi exercise. J Rehabil Med. 2004; 44 (suppl): 12-21.

12. Cieza A, Ewert T, Ustun TB, Chatterji S, Kostanjsek N, Stucki G. Development of ICF Core Sets for patients with chronic conditions. J Rehabil Med. 2004; 44 (suppl): 9-11.

13. Grill E, Ewert T, Chatterji S, Kostanjsek N, Stucki G. ICF Core Sets development for the acute hospital and early post-acute rehabilitation facilities. Disabil Rehabil. 2005; 27: 361-6. 
14. Stucki G, Ustun TB, Melvin J. Applying the ICF for the acute hospital and early post-acute rehabilitation facilities. Disabil Rehabil. 2005; 27: 349-52.

15. Stucki G, Ewert T, Cieza A. Value and application of the ICF in rehabilitation medicine. Disabil Rehabil. 2003; 25: 628-34.

16. Lastra-Martínez I, Martínez-Chamorro M. Funcionamiento, discapacidad y rehabilitación. Inf Psiquiatr. 2004; 175: 19-30.

17. Bruyère $S$, Van Looy S, Peterson D. The International Classification of Function ing, Disability and Health: contemporary literature overview. Rehab Psycol. 2005; 2:121.

18. Jiménez Buñuales MT, González Diego P y Martín Moreno JM. La clasificación internacional del funcionamiento, de la discapacidad y de la salud (CIF) 2001. Rev Esp Salud Pública 2002; 76 (4): 271-279.

19. Ayuso-Mateos JL, Nieto-Moreno M, Sánchez-Moreno J, Vazquez-Barquero JL. Clasificación Internacional del Funcionamiento, la Discapacidad y la Salud (CIF): aplicabilidad y utilidad en la práctica clínica. Med Clin (Barc). 2006; 126 (12): 461-6.

20. Querejeta-González M. Discapacidad/Dependencia. Unificación de criterios de valoración y clasificación. Madrid: Imserso; 2004.

21. Stucki G, Kostanjsek N, Üstün B, Cieza A. ICFbased classification and measurement of functioning. Eur J Phys Rehab Med. 2008; 44: 317-30.

22. Geyh S, Cieza A, Kollerits B, Grimby G, Stucki G. Content comparison of health-related quality of life measures used in stroke based on the international classification of functioning, disability and health (ICF): a systematic review. Qual Life Res. 2007; 16: 833-51.

23. Cieza A, Stucki G. Content comparision of healt related quality of life instruments based on the ICF. Qual Life Res. 2005; 14: 1225-37.

24. Cieza A, Stucki G. The International of Functioning Disability and Health: its development process and content validity. Eur J Phys Rehab Med. 2008; 44: 303-13.

25. Cieza A, Bickenbach J, Chatterji S. The Internation Classification of Functioning Disability and Health as a conceptual platform to specify and discuss health-related concepts. Gesundheitswesen 2008; 70: 47-56.

26. Stucki G, Reinhardt JD, Grimby G, Melvin J. Developing research capacity in human functioning and rehabilitation research from the comprehensive perspective based on the ICF-model. Eur J Phys Rehab Med. 2008; 44: 343-51.

27. Piaget J. The epistemiology of interdisciplinary relationships. In: Apostel L, Berger G, Briggs A, Machaud G, editors. Interdisciplinarity - problems of teaching and research at universities. Paris: OECD; 1972. pp. 127-39.

28. Rosenfield PL. The potencial of transdisciplinary research for sustaining and extending linkages between the health and social sciences. Soc Sci Med. 1992; 35: 1343-57.

29. Frontera WR, Futhrer Mj, Jette AM, Chan L, Cooper RA, Duncan PW et al. editors. Rehabilitation medicine summit: Building research capacity. Am I Phys Med Rehabil. 2005; 84: 913-7.

30. Kuhn TS. The structure of scientific revolutions. Chicago: Universityof Chicago Press; 1962.

31. Stucki G, Reinhardt JD, Cieza A, Brach M, Celio M, Joggi D et al. Developing Swiss paraplegic research: building a research institution from the comprehensive perspective. Disabil Rehabil. 2007; 14: $1-16$.

32. Stucki G, Grimby G, Organizing human functioning and rehabilitation research into distinct scientific fields. Part I: Developing a comprehensive structure from the cell to society. J Rehabil Med. 2007; 39: 293-8.

33. Reinhardt JD, Hofer P, Arenz S, Stucki G. Organizing human functioning and rehabilitation research into distinct scientific fields. Part III: Scientific journals. J Rehabil Med. 2007; 39: 308-22. 
